\title{
Searching for visual companions of close Cepheids
}

\section{VLT/NACO lucky imaging of Y Oph, FF Aql, X Sgr, W Sgr, and $\eta$ AqI ${ }^{\star}$}

\author{
A. Gallenne ${ }^{1}$, P. Kervella ${ }^{2}$, A. Mérand ${ }^{3}$, N. R. Evans ${ }^{4}$, J. H. V. Girard ${ }^{3}$, W. Gieren ${ }^{1}$, and G. Pietrzyński ${ }^{1,5}$ \\ ${ }^{1}$ Universidad de Concepción, Departamento de Astronomía, Casilla 160-C, Concepción, Chile \\ e-mail: agallenne@astro-udec.cl \\ 2 LESIA, Observatoire de Paris, CNRS UMR 8109, UPMC, Université Paris Diderot, 5 place Jules Janssen, 92195 Meudon, France \\ 3 European Southern Observatory, Alonso de Córdova 3107, Casilla 19001, Santiago 19, Chile \\ ${ }^{4}$ Smithsonian Astrophysical Observatory, MS 4, 60 Garden Street, Cambridge MA 02138, USA \\ 5 Warsaw University Observatory, Al. Ujazdowskie 4, 00-478 Warsaw, Poland \\ Received 24 March 2014 / Accepted 13 May 2014
}

\section{ABSTRACT}

\begin{abstract}
Aims. High-resolution imaging in several photometric bands can provide color and astrometric information of the wide-orbit component of Cepheid stars. Such measurements are needed to understand the age and evolution of pulsating stars. In addition, binary Cepheids have the potential to provide direct and model-independent distances and masses.

Methods. We used the NAOS-CONICA adaptive optics instrument (NACO) in the near-infrared to perform a deep search for wide components around the classical Cepheids, Y Oph, FF Aql, X Sgr, W Sgr, and $\eta$ Aql, within a field of view of $1.7^{\prime \prime} \times 1.7^{\prime \prime}\left(3.4^{\prime \prime} \times 3.4^{\prime \prime}\right.$ for $\eta$ Aql).

Results. We were able to reach contrast $\Delta H=5-8 \mathrm{mag}$ and $\Delta K_{\mathrm{s}}=4-7 \mathrm{mag}$ in the radius range $r>0.2^{\prime \prime}$, which enabled us to constrain the presence of wide companions. For Y Oph, FF Aql, X Sgr, W Sgr, and $\eta$ Aql at $r>0.2^{\prime \prime}$, we ruled out the presence of companions with a spectral type that is earlier than a B7V, A9V, A9V, A1V, and G5V star, respectively. For 0.1" $<r<0.2^{\prime \prime}$, no companions earlier than $\mathrm{O} 9 \mathrm{~V}, \mathrm{~B} 3 \mathrm{~V}, \mathrm{~B} 4 \mathrm{~V}, \mathrm{~B} 2 \mathrm{~V}$, and $\mathrm{B} 2 \mathrm{~V}$ star, respectively, are detected. A component is detected close to $\eta$ Aql at projected separation $\rho=654.7 \pm 0.9$ mas and a position angle $\mathrm{PA}=92.8 \pm 0.1^{\circ}$. We estimated its dereddened apparent magnitude to be $m_{\mathrm{H}}^{0}=9.34 \pm 0.04$ and derived a spectral type that ranges between an F1V and an F6V star. Additional photometric and astrometric measurements are necessary to better constrain this star and check its physical association to the $\eta$ Aql system.
\end{abstract}

Key words. instrumentation: adaptive optics - techniques: high angular resolution - stars: variables: Cepheids - binaries: visual

\section{Introduction}

Classical Cepheids are of fundamental importance for the Galactic and extragalactic distance scale. Therefore, a good understanding of their physical properties is necessary. More particularly, their mass and distance need to be known with a good accuracy to better constrain models of pulsating stars. Cepheids in binary systems are unique tools to independently estimate the dynamical mass and distance.

However, companions to Cepheids are hard to detect. Cepheids are bright-supergiant stars, outshining their less evolved companion, which is still on the main sequence. In addition, the small angular separation of most of the companions with respect to the Cepheid ( $\sim 1-50$ mas) makes their spatial resolution difficult with single-dish telescopes. So far, only four (wide) companions have been imaged with the Hubble Space Telescope (HST; Polaris, $\eta$ Aql, V659 Cen and S Nor, Evans et al. 2008, 2013) ; otherwise, the presence of the companions are generally revealed from UV spectra (e.g. Evans 1992) or radial velocity (RV) measurements (e.g. Szabados 1991). Recent examples of the effectiveness of the RV method to detect Cepheids in binary systems are the discoveries of three Cepheids in eclipsing binary systems in the Large Magellanic Cloud (Pietrzyński et al. 2010, 2011; Pilecki et al. 2013; Gieren et al. 2014). Imaging and RVs are complementary techniques. Indeed, only close companions have a notable effect on the

\footnotetext{
^ Based on observations made with ESO telescopes at Paranal Observatory under program ID 089.D-0040.
}

Cepheid RVs, while wide components can be detected from imaging. The second difficulty lies in the brightness difference between the two stars. Most of the companions are hot mainsequence stars ; therefore the brightness of Cepheids makes their detection difficult for wavelengths longer than $0.5 \mu \mathrm{m}$.

Recently, Gallenne et al. (2013c,b) used multi-telescope interferometry to spatially resolve the companions located at a few milli-arcseconds (mas) from the Cepheids. Astrometric measurements combined with spectroscopy were used to determine all the orbital elements of the V1334 Cyg system. However, RV measurements of the secondary are still missing to enable mass and distance estimates. Although interferometry is complementary to the previous mentioned techniques that probe spatial scales $<50$ mas (see also Gallenne et al. 2013a), it is still limited to bright companions (typically $\Delta H \lesssim 5-6$ ).

We report new observations of five Classical Cepheids using adaptive optic (AO) imaging in two broad- and two narrow-band filters. These observations allowed us to search for companions inside $1.7^{\prime \prime} \times 1.7^{\prime \prime}$ and $3.4^{\prime \prime} \times 3.4^{\prime \prime}$ field of views (FoV). The observations and data reduction procedure are detailed in Sect. 2. We then describe the method we used to search for companions in Sects. 3 and 4. Astrometric and photometric measurements of the companion of $\eta \mathrm{Aql}$ is detailed in Sect. 5. Our results are then discussed in Sect. 6.

\section{Observation and data reduction}

The Cepheids were observed with the NACO instrument installed at the Nasmyth B focus of UT4 of ESO VLT. This is an 
Table 1. Log of our NACO observations.

\begin{tabular}{|c|c|c|c|c|c|c|c|c|c|c|c|}
\hline \# & JD & Star & $\phi$ & Filter $^{a}$ & FoV & $\begin{array}{l}\text { DIT } \\
(\mathrm{ms})\end{array}$ & $N$ & $\begin{array}{l}\text { Seeing }_{\lambda} \\
\left({ }^{\prime \prime}\right)\end{array}$ & $A M$ & $\begin{array}{c}r_{0} \\
(\mathrm{~cm})\end{array}$ & $\begin{array}{c}t_{0} \\
(\mathrm{~ms})\end{array}$ \\
\hline 1 & 2456126.5747 & Y Oph & 0.31 & NB1.64 & $1.7^{\prime \prime} \times 1.7^{\prime \prime}$ & 16 & 3000 & 0.65 & 1.09 & 19.1 & 6.4 \\
\hline 2 & 2456126.5749 & Y Oph & 0.31 & $H$ & $1.7^{\prime \prime} \times 1.7^{\prime \prime}$ & 16 & 3000 & 63 & 1.09 & 19.6 & 6.4 \\
\hline 3 & 2456126.5766 & Y Oph & 0.31 & $N B 2.17$ & $1.7^{\prime \prime} \times 1.7^{\prime \prime}$ & 16 & 3000 & .58 & 1.09 & 20.3 & 6.5 \\
\hline 4 & 2456126.5775 & Y Oph & 0.31 & $K \mathrm{~s}$ & $1.7^{\prime \prime} \times 1.7^{\prime \prime}$ & 16 & 3000 & 0.59 & 1.09 & 20.0 & 6.1 \\
\hline 5 & 2456126.5827 & HD 159527 & - & NB1.64 & $1.7^{\prime \prime} \times 1.7^{\prime \prime}$ & 16 & 3000 & 0.59 & 1.03 & 22.2 & 8.9 \\
\hline 6 & 2456126.5836 & HD 159527 & - & $H$ & $1.7^{\prime \prime} \times 1.7^{\prime \prime}$ & 16 & 3000 & 0.60 & 1.02 & 23.1 & 10.1 \\
\hline 7 & 2456126.5846 & HD 159527 & - & $N B 2.17$ & $1.7^{\prime \prime} \times 1.7^{\prime \prime}$ & 16 & 3000 & 0.56 & 1.02 & 22.4 & 10.6 \\
\hline 8 & 2456126.5855 & HD 159527 & - & $K \mathrm{~s}$ & $1.7^{\prime \prime} \times 1.7^{\prime \prime}$ & 16 & 3000 & 0.57 & 1.02 & 22.6 & 11.0 \\
\hline 9 & 2456127.7393 & FF Aql & 0.66 & NB1.64 & $1.7^{\prime \prime} \times 1.7^{\prime \prime}$ & 16 & 3000 & 0.88 & 1.57 & 12.9 & 4.4 \\
\hline 10 & 2456127.7402 & FF Aql & 0.66 & $H$ & $1.7^{\prime \prime} \times 1.7^{\prime \prime}$ & 16 & 3000 & 0.83 & 1.57 & 12.7 & 4.2 \\
\hline 11 & 2456127.7411 & FF Aql & 0.66 & $N B 2.17$ & $1.7^{\prime \prime} \times 1.7^{\prime \prime}$ & 16 & 3000 & 0.7 & 1.58 & 8 & 4.0 \\
\hline 12 & 2456127.7420 & FF Aql & 0.66 & $K \mathrm{~s}$ & $1.7^{\prime \prime} \times 1.7^{\prime \prime}$ & 16 & 3000 & 0.78 & 1.58 & 12.6 & 3.8 \\
\hline 13 & 2456127.7473 & HD 175743 & - & NB1.64 & $1.7^{\prime \prime} \times 1.7^{\prime \prime}$ & 16 & 3000 & 0.60 & 1.65 & 10.9 & 4.1 \\
\hline 14 & 24561 & HD $175^{\circ}$ & - & $H$ & & 16 & 000 & 0 & 1.66 & 9 & 4.1 \\
\hline 15 & 245 & $\mathrm{HI}$ & - & $N B 2.17$ & & 16 & 00 & 0.5 & 1.67 & & 4.4 \\
\hline 16 & 245 & HD 1757 & - & $K \mathrm{~s}$ & & 1 & 0 & 0 & 1.68 & & 4.4 \\
\hline 17 & 24 & $\eta \mathrm{Aql}$ & 0.97 & NB1.64 & & 3 & 0 & 1.0 & 1.40 & & 4.8 \\
\hline 18 & 245 & $\eta$ Aql & 0.97 & $H$ & 3. & 3 & 2000 & 1.0 & 1.41 & 2 & 4.7 \\
\hline 19 & 2456 & $\eta \mathrm{Aql}$ & 0.97 & NB2.17 & 3.4 & 3 & 2000 & 0.9 & 1.42 & 5 & 4.4 \\
\hline 20 & 2456128.8025 & $\eta \mathrm{Aql}$ & 0.97 & $K \mathrm{~s}$ & $3.4^{\prime \prime} \times 3.4^{\prime \prime}$ & 39 & 2000 & 0.9 & 1.43 & 12.4 & 4.4 \\
\hline 21 & 2456128.8067 & HD 188512 & - & $N B 1.64$ & $3.4^{\prime \prime} \times 3.4^{\prime \prime}$ & 35 & 2000 & 0.6 & 1.54 & 13.2 & 4.8 \\
\hline 22 & 2456128.8079 & HD 1885 & - & $H$ & $3.4^{\prime \prime} \times 3.4^{\prime \prime}$ & $3 \mathrm{~s}$ & 2000 & 0.7 & 1.55 & 13.1 & 4.8 \\
\hline 23 & 2456128.8092 & HD 1885 & - & $N B 2.17$ & $3.4^{\prime \prime} \times 3.4^{\prime \prime}$ & 36 & 2000 & 0.6 & 1.56 & 8 & 4.7 \\
\hline 24 & 2456128.8104 & HD 18851 & - & $K \mathrm{~s}$ & $3.4^{\prime \prime} \times$ & 36 & 2000 & 0.6 & 1.57 & 8 & 4.7 \\
\hline 25 & 24561 & X Sgr & 0.68 & NB1.64 & $"$ & 16 & 3000 & 0.81 & 1.42 & 17.2 & 8.8 \\
\hline 26 & 2456167.6434 & X Sgr & 0.68 & $H$ & $1.7^{\prime \prime} \times 1.7^{\prime \prime}$ & 16 & 3000 & 0.71 & 1.43 & 17.4 & 8.9 \\
\hline 27 & 2456167.6444 & X Sgr & 0.68 & $N B 2.17$ & $1.7^{\prime \prime} \times 1.7^{\prime \prime}$ & 16 & 3000 & 0.78 & 1.43 & 17.5 & 9.3 \\
\hline 28 & 2456167.6453 & X Sgr & 0.68 & $K \mathrm{~s}$ & & 16 & 3000 & 0.73 & 1.44 & 17.5 & 9.5 \\
\hline 29 & & & & BB1.64 & & 16 & & & 1.54 & & 4.9 \\
\hline 30 & & W Sgr & 0.38 & $H$ & & 16 & 3000 & 0.67 & 1.55 & & 5.2 \\
\hline 31 & 245 & W Sgr & 0.38 & $N B 2.17$ & $1.7^{\prime \prime} \times 1.7^{\prime \prime}$ & 16 & 3000 & 0.66 & 1.56 & & 5.2 \\
\hline 32 & 245 & W Sgr & 0.38 & $K \mathrm{~s}$ & & 16 & 3000 & 0.67 & 1.57 & & 4.9 \\
\hline 33 & 24561 & D 16629 & - & NB1.64 & $1.7^{\prime \prime} \times 1.7^{\prime \prime}$ & 16 & 3000 & 0.87 & 1.65 & 12.7 & 5.2 \\
\hline 34 & 245616 & HD 1662 & - & $H$ & $\times 1.7^{\prime \prime}$ & 16 & 3000 & 0.83 & 1.67 & 13.0 & 5.3 \\
\hline 35 & 245616 & HD 16620 & - & $N B 2.17$ & $1.7^{\prime \prime} \times 1.7^{\prime \prime}$ & 16 & 3000 & 0.71 & 1.68 & 13.0 & 5.6 \\
\hline 36 & 2456167.6845 & HD 166295 & - & $K \mathrm{~s}$ & $1.7^{\prime \prime} \times 1.7^{\prime \prime}$ & 16 & 3000 & 0.77 & 1.69 & 13.2 & 5.6 \\
\hline
\end{tabular}

Notes. $\phi$ : pulsation phase, estimated with the ephemeris from Samus et al. (2009). $N$ : number of frames in the cube. FoV: field-of-view. Seeing : $^{\circ}$ seeing at the observed wavelength, converted from the one measured in $V$ at the spots of the Shack-Hartmann active optics wavefront sensor. AM: mean airmass. $r_{0}, t_{0}$ : average values of the Fried parameter and coherence time, estimated from the adaptive-optics real time calculator. ${ }^{(a)}$ All broad-band observations were used with a neutral density filter.

adaptive optics system (Rousset et al. 2003, NAOS) and a highresolution near-IR camera (Lenzen et al. 2003, CONICA), which works as an imager or as a spectrograph in the range $1-5 \mu \mathrm{m}$. We used the S13 camera (FoV of $\left.13.5^{\prime \prime} \times 13.5^{\prime \prime}\right)$ with the $H$ and $K \mathrm{~s}$ broadband filters $\left(\lambda_{0}=1.66 \mu \mathrm{m}, \Delta \lambda=0.33 \mu \mathrm{m}\right.$ and $\lambda_{0}=2.18 \mu \mathrm{m}, \Delta \lambda=0.35 \mu \mathrm{m}$, respectively), and the narrow band filters $N B 1.64\left(\lambda_{0}=1.644 \mu \mathrm{m}, \Delta \lambda=0.018 \mu \mathrm{m}\right)$ and $N B 2.17$ $\left(\lambda_{0}=2.166 \mu \mathrm{m}, \Delta \lambda=0.023 \mu \mathrm{m}\right)$. We chose the cube mode which allows us to record very short exposures, and reach truly diffraction-limited images. The efficiency of the cube mode vs. the standard long exposure mode is discussed in Kervella et al. (2009). A gain of $9 \%$ in Strehl ratio with respect to a long exposure has been demonstrated by Girard et al. (2010) in the $K$ band. For all observations in broadband, the neutral density filter ND_Short (reducing the intensity by a factor of 80) was used due to the target brightness.

Data were obtained in July and August 2012 with a window size of $128 \times 130$ pixels, and the shortest allowed exposure time of $16 \mathrm{~ms}$, except for $\eta$ Aql for which a window size of $256 \times 258$ pixels and $39 \mathrm{~ms}$ exposure time were used. The $\log$ of our observations is presented in Table 1 . The point spread function (PSF) calibrator stars were observed immediately after the Cepheids with the same instrumental configurations, except for X Sgr, for which the chosen calibrator had moved out too quickly from the FoV, for an unknown technical reason. For this star, we used the calibrator of W Sgr observed one hour later.

Each raw image in the cube was processed in a standard way, using bias subtraction, flat-field, and bad pixel corrections. The negligible sky background was not subtracted. We then carried out a precentering and a sorting according to the maximum intensity of the central peak. We then applied the shift-and-add technique (Bates \& Cady 1980) to obtain the best possible angular resolution. This method enables to enhance the Strehl ratio by selecting the frames that are the least altered by the atmospheric turbulence. When used with adaptive optics, this technique also reduces the halo contribution (i.e the residual light out of the coherent core) by selecting the best AO-corrected frames. Our processing steps were as follows. We first selected the top $10 \%$ of the frames with the brightest pixel (as a tracer of the Strehl ratio) in our data cubes. We then spatially resampled them by a factor of 4 using a cubic spline interpolation and co-aligned them using a Gaussian fitting on the central core at a precision level of a few 

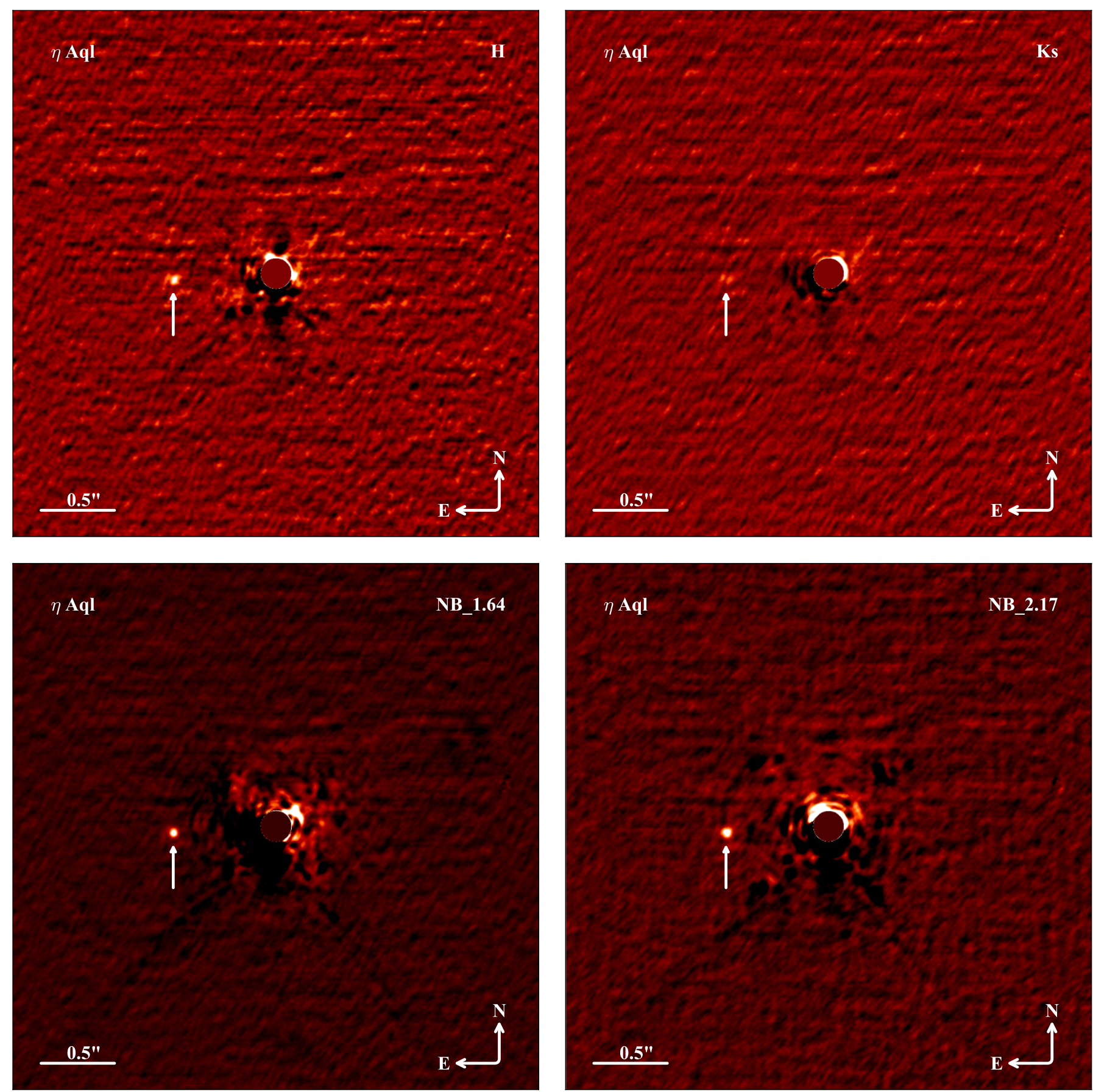

Fig. 1. PSF-subtracted images of $\eta$ Aql. The position of the companion is indicated with the white arrow.

mas (this method is described in detail in Kervella et al. 2009). Each cube was then averaged to obtain the final mean images.

\section{PSF subtraction}

For each final Cepheid image, we subtracted the corresponding PSF-reference image to remove most of the contribution from the central part. The classical PSF-subtraction technique significantly enhanced the capability to detect companions. We first recentered the PSF image, according to the Cepheid image using a cubic spline interpolation, and then scaled the PSF flux with respect to the Cepheid for $0<r<0.1^{\prime \prime}(\sim 2 \lambda / D)$. We therefore expect to detect the companions farther than $0.1^{\prime \prime}$. We finally cleaned the images by removing the correlated noise (correlated lines in the background detector) by performing a median subtraction per line and then a ring median subtraction.

The companion orbiting $\eta$ Aql is clearly detected in the PSF-subtracted images shown in Fig. 1, although some artefacts (spider's pattern, etc.) are still present due to an imperfect PSF subtraction. Because of the neutral density filter, the companion appears brighter in the narrow band filters.

For the other Cepheids, no companion is detected, which might mean that no wide component is present or it is too faint in these bands. We can, however, set upper limits on the magnitude difference for our observing wavelengths. 

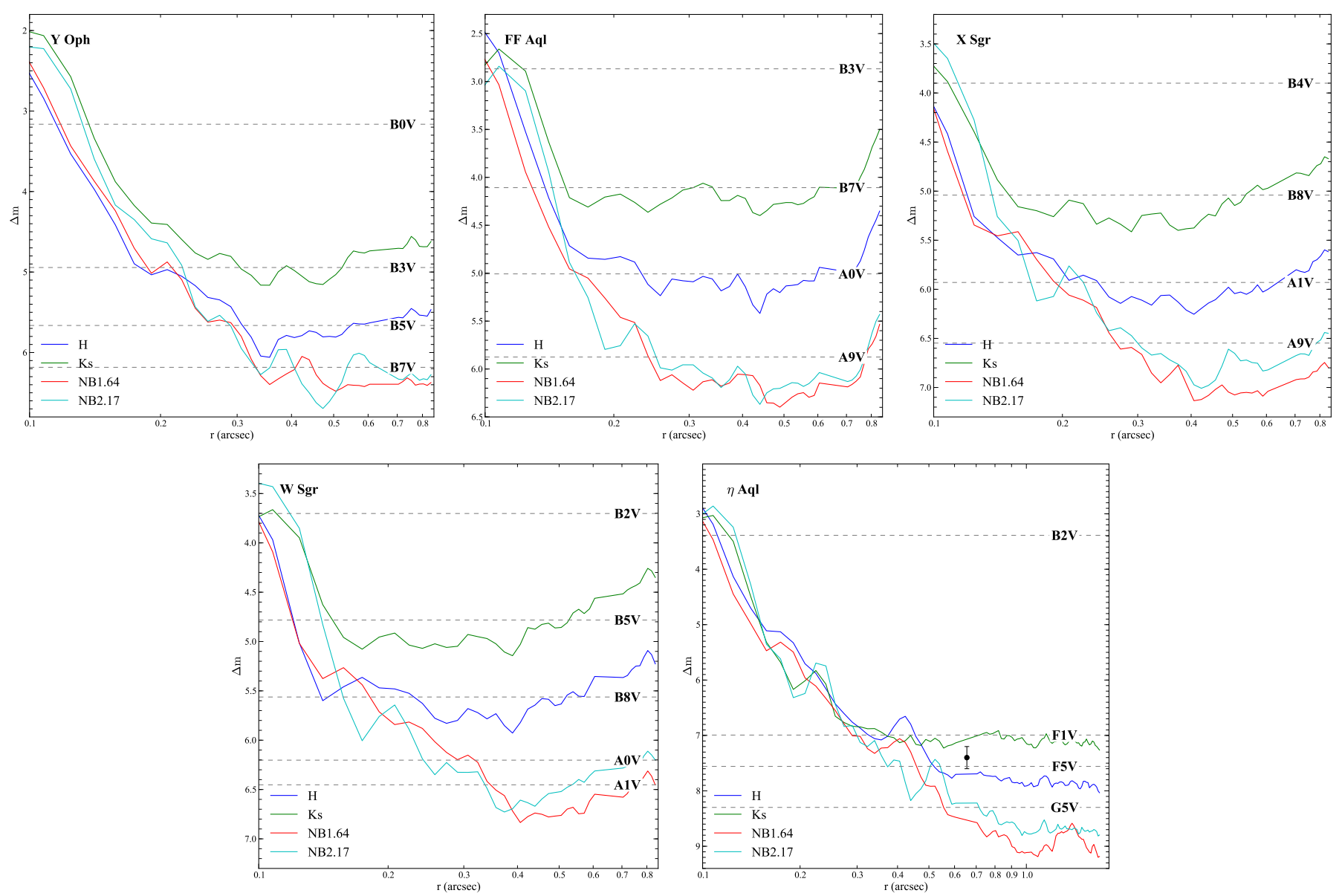

Fig. 2. VLT/NACO $5 \sigma$ detection limits as a function of angular distance for Y Oph, FF Aql, X Sgr, W Sgr and $\eta$ Aql, in all filters. The dot in the $\eta$ Aql plot denotes the measured contrast of the detected companion in the $H$ band.

\section{Detection limits}

For each star, we estimated the $3 \sigma$ detection limit by evaluating the noise within azimuthal rings divided by the primary star maximum flux (Chauvin et al. 2012; Masciadri et al. 2005). The curves are reported in Fig. 2 for all filters. To convert from pixels to angular values, we used the pixel scale $13.26 \pm 0.03$ mas/pixel (Masciadri et al. 2003).

To set an average detection limit, we estimated the radial mean for $r>0.2^{\prime \prime}$ for each filter, and we used the standard deviation as uncertainty. We also interpolated with a periodic cubic spline function the $H$ and $K$ band light curves available in the literature to estimate the Cepheid magnitude at our given phase and determine the companion magnitude limits.

\subsection{YOph}

The binary nature of this Cepheid was first detected by Abt \& Levy (1978) from RV measurements with an orbital period of 2612 days. Pel (1978) found that the star is too blue for its pulsation period in the $(V-B)$ color and suspected a possible blue companion. However, Evans (1992) did not detect it in the IUE spectra and set an upper limit for the companion spectral type of A0V star. Szabados (1989) gathered all RVs and revised the orbital period to 1222.5 days. The companion was also not detected by Hartkopf \& McAlister (1984) from speckle interferometry, leading to a magnitude difference $\Delta m_{V}>2$ for projected separations $>30$ mas.

From our NACO images, no companions are detected. Figure 2 shows that the companion has to be cooler than a
B6V star if it is located at $r>0.2^{\prime \prime}$. We derived the contrast limits, $\Delta H>5.6 \pm 0.2 \mathrm{mag}$ and $\Delta K \mathrm{~s}>4.8 \pm 0.2 \mathrm{mag}$ for $r>0.2^{\prime \prime}$. In the narrow-band filters, we estimated a dynamic range higher than $6.1 \pm 0.2 \mathrm{mag}$ for the same radius range. Using the $H$ and $K$ magnitudes from Laney \& Stobie (1992), this translates to a magnitude limit for the companion of $m_{\mathrm{H}}>8.4 \pm 0.2 \mathrm{mag}$ and $m_{K \mathrm{~s}}>7.5 \pm 0.5 \mathrm{mag}$. For $0.1^{\prime \prime}<r<0.2^{\prime \prime}$, we can safely derive $\Delta H \simeq \Delta K>2.5 \mathrm{mag}$, which leads to $m_{\mathrm{H}}>5.3 \mathrm{mag}$ and $m_{K \mathrm{~s}}>5.1 \mathrm{mag}$.

\subsection{FF Aql}

This is a possible quadruple system with a visual component. The spectroscopic companion was first detected by Abt (1959) from RV measurements, although the total amplitude of the $\gamma$-velocity is small. Szabados (1977) also explained the scatter in the O-C RV diagram as due to orbital motion. Evans et al. (1990) performed a complete study of this system and deduced the orbital elements by combining new and old RV measurements to derive an orbital period of 1430 days. Benedict et al. (2007) included orbital perturbations to their HST parallax fit and derived all the orbital elements of the spectroscopic system (semimajor axis of 12.8 mas).

Wider companions are, however, more uncertain. McAlister et al. $(1987,1989)$ resolved a companion at two different epoch, but with two inconsistent separations $\left(0.15^{\prime \prime}\right.$ and $\left.0.23^{\prime \prime}\right)$ for a same position angle (PA). There are still no proofs about a physical association. Jeffers et al. (1963) reported the detection of an additional visual component at separations ranging $6.3^{\prime \prime}-6.8^{\prime \prime}$ 
with PA from $132^{\circ}$ to $141^{\circ}$ (from 1886 to 1959 ). Recently from adaptive optics imaging, however, Roberts (2011) also detected the component at $6.8^{\prime \prime}$ with a similar PA (144' in 2002). There are three possible explanations. First, this system might have an orbital period of about $43 \mathrm{yr}$; however, this would not be consistent with the minimum period. Indeed, by using Kepler's law of a triple system (Cepheid+spectroscopic+wide companion, we supposed the speckle one does not exist), assuming the measured projected separation $6.8^{\prime \prime}$ is a lower limit for the angular semimajor axis, a distance $d=356$ pc (Benedict et al. 2007), and taking a total mass of about $10 M_{\odot}$ (assuming a mass ratio of unity for each component with respect to the Cepheid and with $3.2 M_{\odot}$ for FF Aql, see Sect. 6), the minimal orbital period would be $\sim 100 \mathrm{yr}$. The second explanation would be that the component did not move from 6.8", meaning that the orbital period would be longer than $43 \mathrm{yr}$, which is even longer than the period limit. The last explanation would be that this star did not move because it is not physically bound to the system. This was also the conclusion of Evans et al. (1990), who detected a companion with a spectral type between A9V and F3V from IUE spectra and concluded that it is the spectroscopic one.

In our observations, the visual component is out of the NACO FoV; the spectroscopic one is inside the PSF and cannot be detected, and we did not detect the speckle companion. For $r>0.2^{\prime \prime}$, we derived average upper limits of $\Delta H>5.0 \pm 0.2 \mathrm{mag}$ and $\Delta K \mathrm{~s}>4.1 \pm 0.2 \mathrm{mag}$, which convert to $m_{\mathrm{H}}>8.6 \pm 0.2 \mathrm{mag}$ and $m_{K \mathrm{~s}}>7.6 \pm 0.2 \mathrm{mag}$, using the light curves from Welch et al. (1984). For NB1.64 and NB2.17, we estimated a magnitude difference larger than $6.0 \pm 0.2 \mathrm{mag}$. For $0.1^{\prime \prime}<r<0.2^{\prime \prime}$, we conservatively estimated $\Delta H \simeq \Delta K>2.8 \mathrm{mag}$, which leads to $m_{\mathrm{H}}>6.4 \mathrm{mag}$ and $m_{K \mathrm{~s}}>6.3 \mathrm{mag}$.

\section{3. $X S g r$}

A large scatter in the RV data was observed by Lloyd Evans (1968), but he did not suspect a possible orbital effect caused by a companion. Szabados (1990) found that the velocity curve is best fitted by including an orbital period and derived a period of about 507 days. The IUE observations were also carried out on this star by Evans (1992), but they did not detect the companion. They, however, set an upper limit for the spectral type of A0V. The binary nature was also analysed by Groenewegen (2008) and derived a slightly longer orbital period of about 574 days.

More recently, using the AMBER interferometric recombiner, Li Causi et al. (2013) reported the detection of the companion at a projected separation of 10.7 mas with a flux ratio of about $0.6 \%$; however, such a result has to be confirmed as this detection is at the capability limits of the instrument (see e.g. Absil et al. 2010)

The spectroscopic component is not detectable from our NACO observations, while other wide companions are not detected. We set contrast limits for a wide component at $r>0.2^{\prime \prime}$ of $\Delta H>6.0 \pm 0.2 \mathrm{mag}$ and $\Delta K \mathrm{~s}>5.0 \pm 0.2 \mathrm{mag}$, transforming $m_{\mathrm{H}}>8.7 \pm 0.2 \mathrm{mag}$ and $m_{K \mathrm{~s}}>7.7 \pm 0.2 \mathrm{mag}$ (using the light curves from Feast et al. 2008). We also measured a mean dynamic range higher than $6.6 \pm 0.2 \mathrm{mag}$ in the narrow band filters. For $0.1^{\prime \prime}<r<0.2^{\prime \prime}$, we estimated $\Delta H \simeq \Delta K>3.9$ mag, leading to $m_{\mathrm{H}}>6.7 \mathrm{mag}$ and $m_{K \mathrm{~s}}>6.5 \mathrm{mag}$.

\subsection{W Sgr}

This is a triple system composed of a spectroscopic and a visual component. The multiplicity of this star has been studied by Babel et al. (1989) who derived the first spectroscopic orbital elements of the system, with an orbital period of 1780 days. Benedict et al. (2007) later deduced all the orbital elements from HST observations, including a semimajor axis of 12.9 mas, and a revised period of 1582 days. Evans et al. (2009) constrained the spectral type of this close companion to be later than an F0V star.

The visual companion was first resolved by Morgan et al. (1978) at $\sim 0.12^{\prime \prime}$ from speckle interferometry ; however, it was not detected by Bonneau et al. (1980) with the same observing technique. Then, a hot companion is detected from IUE spectra (Böhm-Vitense \& Proffitt 1985; Evans 1991) with a spectral type A0V, which might be the wide component. Evans et al. (2009) confirmed these results with the detection of both companions with HST STIS with the widest star located at $\sim 0.16^{\prime \prime}$.

This wide component is inside the NACO FoV; however, it is just below our detection limits. We estimated a contrast to be $\Delta H>5.5 \pm 0.2 \mathrm{mag}$ and $\Delta K \mathrm{~s}>4.7 \pm 0.3 \mathrm{mag}$ for $r>0.2^{\prime \prime}$, which convert to $m_{\mathrm{H}}>8.4 \pm 0.2 \mathrm{mag}$ and $m_{K \mathrm{~s}}>7.5 \pm 0.3 \mathrm{mag}$ (using light curves of Welch et al. 1984). We found a contrast higher than $6.4 \pm 0.3 \mathrm{mag}$ in the narrow-band filters. For $0.1^{\prime \prime}<$ $r<0.2^{\prime \prime}$, we estimated $\Delta H \simeq \Delta K>3.7 \mathrm{mag}$, leading to $m_{\mathrm{H}}>$ $6.6 \mathrm{mag}$ and $m_{K \mathrm{~s}}>6.4 \mathrm{mag}$.

\section{5. $\eta \mathrm{Aql}$}

The first evidence of a companion was given from IUE spectra (Mariska et al. 1980), where a significant UV emission corresponding to an A0V star is detected. However, there are no signs of short-term orbital effects in the RV measurements, which would be consistent with a wide component with low velocity amplitude. Additional IUE spectra later confirmed the presence of a hot companion near $\eta$ Aql (Evans 1991) and refined the spectral type to be B9.8V. However, this component was not detected from speckle interferometry (Mason et al. 1999) with a typical resolution limit of 40 mas. No detection was also reported from the Hipparcos telescope (Mason et al. 1999), which set typical detection limits in the $V$ band of $\Delta m=0.8,2.7$, and $4.0 \mathrm{mag}$ for separation $\rho<0.1,0.2$, and $0.5^{\prime \prime}$, respectively. From HST FGS observations, Benedict et al. (2007) found some astrometric perturbations in their measurements, but they were not able to fit an orbital motion because of a lack of constraints from RV variations. Evans et al. (2013) resolved a companion at $0.66^{\prime \prime}$ with the HST. This companion is located too far from the Cepheid to be the one detected by Benedict et al. (2007), and as mentioned by Evans et al. (2013), this would indicate the presence of a third closest component.

A companion is detected in our NACO images, and its position is consistent with the one resolved with HST. The $\eta$ Aql system is analysed more particularly in Sect. 5. We also derived upper magnitude limits to exclude the presence of other companions in our FoV. For $r>0.2^{\prime \prime}$, we estimated $\Delta H>7.6 \pm 0.5 \mathrm{mag}$ and $\Delta K s>7.0 \pm 0.2 \mathrm{mag}$, which convert to $m_{\mathrm{H}}>9.7 \pm 0.5 \mathrm{mag}$ and $m_{K \mathrm{~s}}>9.0 \pm 0.2 \mathrm{mag}$ (using light curves from Barnes et al. 1997). For NB1.64 and NB2.17, we estimated a magnitude difference larger than $8.3 \pm 0.2 \mathrm{mag}$. In the range $0.1^{\prime \prime}<r<0.2^{\prime \prime}$, we evaluated $\Delta H \simeq \Delta K>3.4 \mathrm{mag}$, giving $m_{\mathrm{H}} \sim m_{K \mathrm{~s}}>5.4 \mathrm{mag}$.

\section{The wide component of $\eta$ Aql}

The wide component around $\eta$ Aql is clearly detected in the PSF-subtracted images of Fig. 1, except in the $K$ s band where its detection is marginal. In this section, we estimate its angular position relative to the Cepheid and its magnitude in the 

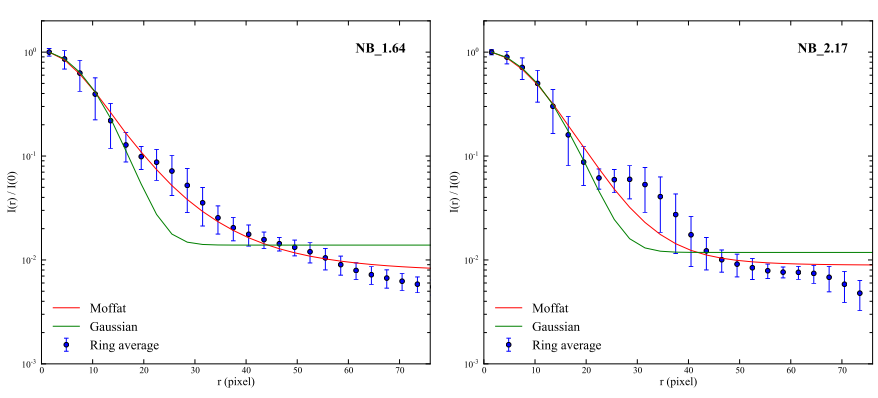

Fig. 3. Radial profile of the $\eta$ Aql images in the narrow band filters. Radial flux was evaluated estimating the average value and standard deviation in rings.

corresponding filters. It is worth mentioning, however, that its physical association to the Cepheid has not been proven yet.

\subsection{Astrometric position}

We estimated the relative position by fitting a 2D-Moffat and 2D-Gaussian function, respectively for the Cepheid and the companion, which have the following form

$$
\begin{aligned}
& I(x, y)_{\text {ceph }}=I_{0, \text { ceph }}\left[\left(\frac{x-x_{0}}{\rho_{x}}\right)^{2}+\left(\frac{y-y_{0}}{\rho_{y}}\right)^{2}+1\right]^{-\beta}+c_{1}, \\
& I(x, y)_{\text {comp }}=I_{0, \text { comp }} \exp \left(-\frac{\left(x-x_{0}\right)^{2}}{2 \sigma_{x}^{2}}-\frac{\left(y-y_{0}\right)^{2}}{2 \sigma_{y}^{2}}\right)+c_{2},
\end{aligned}
$$

where $x_{0}, y_{0}$ are the coordinates of the center of each star, $\left(\rho_{x}, \rho_{y}\right)$ denote the full width at half maximum of the PSF, $\left(\sigma_{x}, \sigma_{y}\right)$ the standard deviations of the Gaussian, $\left(c_{1}, c_{2}\right)$ are constants, and $\beta$ is a variable power law index. We chose a $2 \mathrm{D}$-Moffat function for the Cepheid, because it has the advantage to better fit the AO PSF wings, as shown in Fig 3. Indeed, the PSF wings are more extended because of the residual light out of the coherent core (AO halo) for bright stars.

We extracted subwindows of $76 \times 76$ pixels $\left(0.25^{\prime \prime} \times 0.25^{\prime \prime}\right)$ for the Cepheid and $30 \times 30$ pixels $\left(0.10^{\prime \prime} \times 0.10^{\prime \prime}\right)$ for the companion. We measured the relative position in the narrow band filters $N B 2.17$ and NB1.64, in which the signal-to-noise ratio of the companion is higher. In the broadband filters, the flux of the companion is too low to obtain a consistent fit. Uncertainties were estimated using the bootstrapping technique with 500 bootstrap samples. Enlarging the sub-window has an impact of at most $0.5 \%$ on $\Delta \delta$, which is well below our accuracy level, while it is negligible on $\Delta \alpha$.

Refraction effects by the Earth's atmosphere causes an apparent change in the true astrometric position. These effects depend on the wavelength and the atmospheric column depth. In our case, the chromatic differential refraction has no impact as we used narrow-band filters. However, the achromatic refraction has to be corrected for. We estimated these corrections, following the method of Kervella et al. (2013), which uses the slarefro function distributed with the Starlink library ${ }^{1}$. The right ascension and declination corrections to add to the measured differential astrometry are the values, $\Delta \alpha_{\text {corr }}=171.42 \mu$ as and $\Delta \delta_{\text {corr }}=-130.04 \mu$ as at $2.166 \mu \mathrm{m}$, and $\Delta \alpha_{\text {corr }}=170.94 \mu$ as and $\Delta \delta_{\text {corr }}=-132.41 \mu$ as at $1.644 \mu \mathrm{m}$ (it is worth mentioning that these values are below our accuracy level, i.e. $<0.15 \sigma)$. The

\footnotetext{
1 We actually used the pySLALIB module which contains Python wrappers for every Fortran functions in the SLALIB library.
}

Table 2. Relative astrometric position of the companion of $\eta$ Aql, corrected for atmospheric refraction.

\begin{tabular}{lccc}
\hline \hline Filter & MJD & $\begin{array}{c}\Delta \alpha \\
(\mathrm{mas})\end{array}$ & $\begin{array}{c}\Delta \delta \\
\text { (mas) }\end{array}$ \\
\hline NB1.64 & 56128.2986 & $654.70 \pm 1.16$ & $-32.37 \pm 1.18$ \\
NB2.17 & 56128.3012 & $652.93 \pm 1.24$ & $-30.96 \pm 1.76$ \\
\hline Avg. & 56128.2999 & $653.87 \pm 0.85$ & $-31.93 \pm 0.98$ \\
\hline
\end{tabular}

final astrometric positions are listed in Table 2 with a weighted average value. The values for both filters agree within $1 \sigma$.

The companion is located at an average angular separation $\rho=654.7 \pm 0.9$ mas and a position angle $\mathrm{PA}=92.8 \pm 0.1^{\circ}$. These values are consistent with the detection of Evans et al. (2013, and private communication) using the HST.

\subsection{Photometry}

There is no existing photometric template for HD 188512 ; we, therefore, created a synthetic spectral energy distribution (SED) following the method used by Cohen et al. (1999) and Mérand et al. (2005). We fitted stellar atmosphere models obtained with the ATLAS9 simulation code from Castelli \& Kurucz (2003) for the wavelength range $0.4-25 \mu \mathrm{m}$. We chose a grid that was computed for solar metallicity and a turbulence velocity of $2 \mathrm{~km} \mathrm{~s}^{-1}$. We then interpolated this grid to compute spectra for any effective temperature and any surface gravity. The spectrum was multiplied by the solid angle of the stellar photosphere, $\pi \theta_{\mathrm{LD}} / 4$, where $\theta_{\mathrm{LD}}$ is the limb-darkened angular diameter.

We adjusted the photometric data to the model by taking the spectral response of each instrument into account. We did not adjust the surface gravity, since the broadband photometry is mostly insensitive to this parameter. Therefore, we took an average effective gravity of $\log g=2.0$. Changing this value by \pm 1.0 leads to a variation in the derived parameters of less than $2 \%$, which we also considered in the final uncertainties for the corresponding stars. During the fitting procedure, we also fitted the color excess $E(B-V)$ for all flux densities $<3 \mu \mathrm{m}$ by adopting the reddening law from Fouqué et al. (2007). Data for $\lambda>3 \mu \mathrm{m}$ were not corrected for the interstellar extinction, which we assumed to be negligible.

We used photometry from the Tycho-2 Catalogue (Høg et al. 2000), the Two Micron All Sky Survey (2MASS, Cutri et al. 2003), the Wide-field Infrared Survey Explorer (WISE, Wright et al. 2010), the Infrared Astronomical Satellite (IRAS, Helou \& Walker 1988), and the AKARI satellite IRC point source catalog (Ishihara et al. 2010).

We then carried out a classical aperture photometry to estimate the flux density of both component in each filter. Absolute calibration was done by considering the filter transmission ${ }^{2}$. We chose an aperture of $0.55^{\prime \prime}$ for the central source (Cepheid and reference star) and a sky annulus thickness of $0.2^{\prime \prime}$ from $0.80^{\prime \prime}$ to avoid the companion. For the component photometry, we took an aperture of $0.06^{\prime \prime}$ and a sky annulus of $0.03^{\prime \prime}$ thickness with an internal radius of $0.09^{\prime \prime}$.

We determined dereddened magnitude by adopting the reddening law from Fouqué et al. (2007) with a total-to-selective absorption in the $V$ band of $R_{V}=3.23$ (Sandage et al. 2004) and a color excess $E(B-V)=0.130$ from Fouqué et al. (2007). The dereddened magnitudes are listed in Table 3 . In the $K$ s band, the flux was too low to obtain a reliable estimate.

\footnotetext{
2 Filter transmission profiles are available on the website http://svo2 . cab.inta-csic.es/theory/fps/
} 
Table 3. Measured dereddened magnitudes.

\begin{tabular}{lcccc}
\hline \hline & $\begin{array}{c}H_{0} \\
(\mathrm{mag})\end{array}$ & $\begin{array}{c}m_{0}^{1.64} \\
(\mathrm{mag})\end{array}$ & $\begin{array}{c}K \mathrm{~s}_{0} \\
(\mathrm{mag})\end{array}$ & $\begin{array}{c}m_{0}^{2.17} \\
(\mathrm{mag})\end{array}$ \\
\hline$\eta$ Aql & $1.82_{ \pm 0.04}$ & $1.81_{ \pm 0.04}$ & $1.80_{ \pm 0.04}$ & $1.79_{ \pm 0.04}$ \\
Companion & $9.34_{ \pm 0.04}$ & $9.31_{ \pm 0.04}$ & - & $9.18_{ \pm 0.04}$ \\
\hline
\end{tabular}

Table 4. Magnitude and spectral types limits for the companions for $r>0.2^{\prime \prime}$.

\begin{tabular}{ccccccc}
\hline \hline Star & $\Delta H$ & $m_{\mathrm{H}}$ & $\Delta K$ & $m_{\mathrm{K}}$ & $\Delta N B$ & Sp. Typ. \\
\hline Y Oph & $>5.6$ & $>8.4$ & $>4.8$ & $>7.5$ & $>6.1$ & B7V \\
FF Aql & $>5.0$ & $>8.6$ & $>4.1$ & $>7.6$ & $>6.0$ & A9V \\
X Sgr & $>6.0$ & $>8.7$ & $>5.0$ & $>7.7$ & $>6.6$ & A9V \\
W Sgr & $>5.5$ & $>8.4$ & $>4.7$ & $>7.5$ & $>6.4$ & A1V \\
$\eta$ Aql & $>7.6$ & $>9.7$ & $>7.0$ & $>9.0$ & $>8.3$ & G5V \\
\hline
\end{tabular}

Notes. $\triangle N B$ denotes the contrast limit in the narrow band filters. Sp. Typ. represents the spectral-type upper limit in the narrow band filters.

\section{Discussion}

Using our measured contrast limits in the $H$ and $K$ s bands, we can exclude the presence of companions or set an upper limit on their spectral type with respect to the angular distance from the Cepheid. We utilized the absolute magnitudes of the MK classification in Cox (2000) and the IR intrinsic colors of Ducati et al. (2001) for main-sequence stars. We also took the distance of the Cepheids (and so the system) from Benedict et al. (2007) and Kervella et al. (2004). We can split our analysis in two distance regimes.

\subsection{Case $r>0.2^{\prime \prime}$}

The upper limits for the spectral type (at $3 \sigma$ ) for the undetected companions are shown in Fig. 2. For Y Oph, FF Aql, X Sgr and W Sgr in the $H$ band, we can exclude the presence of companions with a spectral type that appear earlier than B5V, A0V, A1V, and $\mathrm{B} 8 \mathrm{~V}$, respectively. In the narrow band filters, the spectraltype upper limits are $\mathrm{B} 7 \mathrm{~V}, \mathrm{~A} 9 \mathrm{~V}, \mathrm{~A} 9 \mathrm{~V}$, and $\mathrm{A} 1 \mathrm{~V}$, respectively. The spectral-type limits for Y Oph and FF Aql agree with those derived in the literature ( $\mathrm{A} 0 \mathrm{~V}$ and $\mathrm{A} 1 \mathrm{~V}$, respectively, from Evans 1992, see Sect. 4). However, for X Sgr, the previous limit was an A0V star, we can now exclude companions brighter than A9V stars. No companion later than an A1V star is detected for W Sgr in this radius range.

For $\eta \mathrm{Aql}$, we can set an upper limit of an F5V star in $H$ and $\mathrm{G} 5 \mathrm{~V}$ in the narrow band filters. Our observed companion (black dot in Fig. 2) is detected at $7 \sigma$ in the narrow bands, at $3 \sigma$ in $H$, and is almost not detected in $K$. We derived a spectral type for this companion ranging between an F1V and F6V star.

We summarized the magnitudes and spectral type limits in Table 4.

\subsection{Case $0.1^{\prime \prime}<r<0.2^{\prime \prime}$}

We can also set an upper limit on the spectral type of possible companions in that radius range. For Y Oph, FF Aql, X Sgr, W Sgr, and $\eta \mathrm{Aql}$, we did not detect companions with a spectral type that is earlier than an $09 \mathrm{~V}, \mathrm{~B} 3 \mathrm{~V}, \mathrm{~B} 4 \mathrm{~V}, \mathrm{~B} 2 \mathrm{~V}$, and $\mathrm{B} 2 \mathrm{~V}$ star, respectively. The A0V star companion orbiting $\mathrm{W} \mathrm{Sgr,} \mathrm{which} \mathrm{is}$ located at $\sim 0.15-0.20^{\prime \prime}$, is just below our $3 \sigma$ threshold and is not detected.

In this radius range, we cannot have more constraints, and the detection of fainter companions is limited to the AO halo, which is also the residual light out of the coherent core.

\subsection{Detected component}

The component around $\eta$ Aql is bright and wide enough to be spatially resolve. Its projected separation and position angle are consistent with the component detected by Evans et al. (2013). However, our estimated spectral type, which is between an F1V and F6V star, does not agree with the B9.8 star detected from UV spectra (Evans 1991). On the other hand, Mason et al. (1999) did not detect a component earlier than an A1V star by speckle interferometry at such a separation. Therefore, the B9.8 star should be in a closer orbit, and our detected component, if bound to the system, would be another companion. Additional photometric observations in various other bands are needed to fully constrain its spectral type, while more astrometric measurements will provide information about its association with the $\eta$ Aql system.

\section{Conclusion}

We presented high angular resolution imaging with VLT/NACO to search for wide components around five classical Cepheids. We detected a component close to $\eta \mathrm{Aql}$ at a separation of $0.65^{\prime \prime}$ with a spectral type between F1V and F6V. We measured its dereddened apparent magnitude in the infrared to be $H_{0}=$ 9.34 mag. No other companion is present in the FoV.

In the range $r>0.2^{\prime \prime}$, we ruled out the presence of companions that come earlier than B7V, A9V, A9V, A1V, and G5V, respectively for Y Oph, FF Aql, X Sgr, W Sgr and $\eta$ Aql.

The AO imaging is an useful tool to search for Cepheid companions because it reaches high detection limits. Other techniques, such as differential imaging, aperture masking or coronography, also have the capabilities to detect faint companions. We will be able to reach higher contrasts with the next generation instruments, such as VLT/SPHERE or Gemini/GPI.

Studying Cepheid companions is particularly important to understand the evolution of the pulsating star. Binary Cepheids are also powerful tools in estimating the masses and distances with a unique accuracy. Gaia will revolutionize this field by providing a micro-arcsecond astrometric precisions, and will give accurate Cepheid distances and orbital perturbations.

Acknowledgements. A.G. acknowledges support from FONDECYT grant 3130361. W.G. an G.P. gratefully acknowledge financial support for this work from the BASAL Centro de Astrofísica y Tecnologías Afines (CATA) PFB06/2007. Support from the Polish National Science Center grant MAESTRO 2012/06/A/ST9/00269 and the Polish Ministry of Science grant Ideas Plus (awarded to G.P.) is also acknowledged. We acknowledge financial support from the "Programme National de Physique Stellaire" (PNPS) of CNRS/INSU, France, and the ECOS/Conicyt grant C13U01. This research received the support of PHASE, the high angular resolution partnership between ONERA, the Observatoire de Paris, CNRS, and University Denis Diderot Paris 7. This work made use of the SIMBAD and VizieR astrophysical database from the CDS, Strasbourg, France and the bibliographic informations from the NASA Astrophysics Data System. Data processing for this work has been done using the Yorick language which is freely available at http://yorick. sourceforge. net/

\section{References}

Absil, O., Le Bouquin, J.-B., Lebreton, J., et al. 2010, A\&A, 520, L2 Abt, H. A. 1959, ApJ, 130, 769 
Abt, H. A., \& Levy, S. G. 1978, PASP, 90, 188

Babel, J., Burki, G., Mayor, M., Chmielewski, Y., \& Waelkens, C. 1989, A\&A, 216, 125

Barnes, III, T. G., Fernley, J. A., Frueh, M. L., et al. 1997, PASP, 109, 645

Bates, R. H. T., \& Cady, F. M. 1980, Optics Commun., 32, 365

Benedict, G. F., McArthur, B. E., Feast, M. W., et al. 2007, AJ, 133, 1810

Böhm-Vitense, E., \& Proffitt, C. 1985, ApJ, 296, 175

Bonneau, D., Blazit, A., Foy, R., \& Labeyrie, A. 1980, A\&AS, 42, 185

Castelli, F., \& Kurucz, R. L. 2003, in Modelling of Stellar Atmospheres, eds.

N. Piskunov, W. W. Weiss, \& D. F. Gray (ASP), IAU Symp., 210, A20

Chauvin, G., Faherty, J., Boccaletti, A., et al. 2012, A\&A, 548, A33

Cohen, M., Walker, R. G., Carter, B., et al. 1999, AJ, 117, 1864

Cox, A. N. 2000, Allen's astrophysical quantities, 4th edn (New York: AIP Press)

Cutri, R. M., Skrutskie, M. F., van Dyk, S., et al. 2003, VizieR Online Data Catalog: II/246

Ducati, J. R., Bevilacqua, C. M., Rembold, S. B., \& Ribeiro, D. 2001, ApJ, 558, 309

Evans, N. R. 1991, ApJ, 372, 597

Evans, N. R. 1992, ApJ, 384, 220

Evans, N. R., Welch, D. L., Scarfe, C. D., \& Teays, T. J. 1990, AJ, 99, 1598

Evans, N. R., Schaefer, G. H., Bond, H. E., et al. 2008, AJ, 136, 1137

Evans, N. R., Massa, D., \& Proffitt, C. 2009, AJ, 137, 3700

Evans, N. R., Bond, H. E., Schaefer, G. H., et al. 2013, AJ, 146, 93

Feast, M. W., Laney, C. D., Kinman, T. D., van Leeuwen, F., \& Whitelock, P. A. 2008, MNRAS, 386, 2115

Fouqué, P., Arriagada, P., Storm, J., et al. 2007, A\&A, 476, 73

Gallenne, A., Kervella, P., Mérand, A., et al. 2013a, in EAS Pub. Ser., eds.

A. Pavlovski, K., Tkachenko, \& G. Torres, 64, 197

Gallenne, A., Mérand, A., Kervella, P., et al. 2013b, A\&A, 558, A140

Gallenne, A., Monnier, J. D., Mérand, A., et al. 2013c, A\&A, 552, A21

Gieren, W., Pilecki, B., Pietrzyński, G., et al. 2014, ApJ, 786, 80

Girard, J. H. V., Kasper, M., Quanz, S. P., et al. 2010, in Proc. SPIE, 7736, 2N

Groenewegen, M. A. T. 2008, A\&A, 488, 25

Hartkopf, W. I., \& McAlister, H. A. 1984, PASP, 96, 105

Helou, G., \& Walker, D. W. 1988, IRAS catalogue, Vol. 7 (STI)

Høg, E., Fabricius, C., Makarov, V. V., et al. 2000, A\&A, 355, L27

Ishihara, D., Onaka, T., Kataza, H., et al. 2010, A\&A, 514, A1

Jeffers, H. M., van den Bos, W. H., \& Greeby, F. M. 1963, Index Catalogue of

Visual Double Stars, 1961.0 (Publications of the Lick Observatory, Mount Hamilton)
Kervella, P., Nardetto, N., Bersier, D., Mourard, D., \& Coudé du Foresto, V. 2004, A\&A, 416, 941

Kervella, P., Verhoelst, T., Ridgway, S. T., et al. 2009, A\&A, 504, 115

Kervella, P., Mérand, A., Petr-Gotzens, M. G., Pribulla, T., \& Thévenin, F. 2013, A\&A, 552, A18

Laney, C. D., \& Stobie, R. S. 1992, A\&AS, 93, 93

Lenzen, R., Hartung, M., Brandner, W., et al. 2003, in Proc. SPIE, eds. M. Iye, \& A. F. M. Moorwood, 4841, 944

Li Causi, G., Antoniucci, S., Bono, G., et al. 2013, A\&A, 549, A64

Lloyd Evans, T. 1968, MNRAS, 141, 109

Mariska, J. T., Doschek, G. A., \& Feldman, U. 1980, ApJ, 238, L87

Masciadri, E., Brandner, W., Bouy, H., et al. 2003, A\&A, 411, 157

Masciadri, E., Mundt, R., Henning, T., Alvarez, C., \& Barrado y Navascués, D. 2005, ApJ, 625, 1004

Mason, B. D., Martin, C., Hartkopf, W. I., et al. 1999, AJ, 117, 1890

McAlister, H. A., Hartkopf, W. I., Hutter, D. J., \& Franz, O. G. 1987, AJ, 93, 688

McAlister, H. A., Hartkopf, W. I., Sowell, J. R., Dombrowski, E. G., \& Franz, O. G. 1989, AJ, 97, 510

Mérand, A., Bordé, P., \& Coudé Du Foresto, V. 2005, A\&A, 433, 1155

Morgan, B. L., Beddoes, D. R., Scaddan, R. J., \& Dainty, J. C. 1978, MNRAS, 183,701

Pel, J. W. 1978, A\&A, 62, 75

Pietrzyński, G., Thompson, I. B., Gieren, W., et al. 2010, Nature, 468, 542

Pietrzyński, G., Thompson, I. B., Graczyk, D., et al. 2011, ApJ, 742, L20

Pilecki, B., Graczyk, D., Pietrzyński, G., et al. 2013, MNRAS, 436, 953

Roberts, Jr., L. C. 2011, MNRAS, 413, 1200

Rousset, G., Lacombe, F., Puget, P., et al. 2003, in Proc. SPIE, eds. P. L. Wizinowich, \& D. Bonaccini, 4839, 140

Samus, N. N., Durlevich, O. V., et al. 2009, VizieR Online Data Catalog: B/gcvs. Originally published in: Institute of Astronomy of Russian Academy of Sciences and Sternberg State Astronomical Institute of the Moscow State University, 1, 2025

Sandage, A., Tammann, G. A., \& Reindl, B. 2004, A\&A, 424, 43

Szabados, L. 1977, Commun. of the Konkoly Observatory Hungary, 70, 1

Szabados, L. 1989, Commun. of the Konkoly Observatory Hungary, 94, 1

Szabados, L. 1990, MNRAS, 242, 285

Szabados, L. 1991, Commun. of the Konkoly Observatory Hungary, 96, 123

Welch, D. L., Wieland, F., McAlary, C. W., et al. 1984, ApJS, 54, 547

Wright, E. L., Eisenhardt, P. R. M., Mainzer, A. K., et al. 2010, AJ, 140, 1868 